\title{
The phantom menaced: constraints on low-energy effective ghosts
}

\author{
James M. Cline, Sangyong Jeon, Guy D. Moore \\ Physics Department, McGill University, 3600 University Street, Montréal, Québec, Canada H3A 2T8 \\ e-mail: ghostbusters@physics.mcgill.ca
}

(Dated: November, 2003)

\begin{abstract}
It has been suggested that a scalar field with negative kinetic energy, or "ghost," could be the source of the observed late-time cosmological acceleration. Naively, such theories should be ruled out by the catastrophic quantum instability of the vacuum. We derive phenomenological bounds on the Lorentz-violating ultraviolet cutoff $\Lambda$ which must apply to low-energy effective theories of ghosts, in order to keep the instability at unobservable levels. Assuming only that ghosts interact at least gravitationally, we show that $\Lambda \lesssim 3 \mathrm{MeV}$ for consistency with the cosmic gamma ray background. We also show that theories of ghosts with a Lorentz-conserving cutoff are completely excluded.
\end{abstract}

PACS numbers: $98.80 . \mathrm{Cq}, 98.70 . \mathrm{Vc}$

The present accelerated expansion of the universe seems to be an experimental fact, now that data from distant type Ia supernovae [1] have been corroborated by those from the cosmic microwave background [2]. Although the simplest explanation is a cosmological constant $\Lambda$ of order $\left(10^{-3} \mathrm{eV}\right)^{4}$, this tiny energy scale is so far below the expected "natural" size for a cosmological constant, that alternative explanations have been vigorously pursued. A common approach has been to assume that the true value of $\Lambda$ is zero, due to an unknown mechanism, and to propose new physics which would explain why the present-day vacuum energy differs from zero by the small observed amount.

The most popular idea has been quintessence, in which the universe is gradually approaching the zero of the vacuum energy by the slow rolling of an extremely weakly coupled scalar field. More recently, some less conventional alternatives have been considered, including "phantom matter," which is essentially quintessence with a wrong-sign kinetic term [3]. These models are motivated by the supernova data, which suggest that the dark energy equation of state violates the weak energy condition by having $p<-\rho[\underline{4}]$.

A serious problem with phantom matter, which is overlooked in the literature that attempts to apply it to cosmology, is that such theories are not quantum mechanically viable, either because they violate conservation of probability, or they have unboundedly negative energy density and lead to the absence of a stable vacuum state. Whether a ghost carries negative norm and positive energy, or vice versa, is a choice which is made during the quantization procedure. This choice exists because the $i \epsilon$ prescription for defining the propagator near its poles is not unique, and not specified by the Lagrangian itself. The momentum space propagator for a ghost can have either of the two forms

$$
\frac{-i}{p^{2}-m^{2}+i \epsilon} \quad \text { or } \quad \frac{-i}{p^{2}-m^{2}-i \epsilon}
$$

In the first form in (11), the imaginary part of the propagator has the opposite sign relative to that of a positive norm particle. This will cause the optical theorem to be violated, leading to a nonunitary theory. That is, this choice gives a theory with no probabilistic interpretation. It is therefore unphysical and should be dismissed.

On the other hand, if the second form in (11) is chosen, unitarity is maintained. The price to be paid is that the poles in the propagator are shifted in such a way that particles with negative energy are the ones which propagate forward in time, so ghosts possess negative energy. This means, for instance, that a two-body scattering process involving nonghosts and ghosts can result in an increase in the magnitude of the energies of the particles. To illustrate this, suppose that the ghost is massive so that we can consider it to be initially at rest. If the initial energy of a photon is $E_{i}$ and it gravitationally scatters from the ghost at angle $\theta$, then its final energy is

$$
E_{f}=E_{i}\left(\frac{m}{m-E_{i}(1-\cos \theta)}\right)>E_{i}
$$

in contrast to the nonghost case where photons can only lose energy in such scatterings. In fact, there exist initial energies $E_{i}=m /(1-\cos \theta)$ such that the final energy is divergent. The final energy of the ghost is correspondingly large and negative.

To avoid this kind of problem, one should consider theories where the interactions between ghosts and normal matter are as weak as possible. However, we must allow the ghosts to interact gravitationally, since it is their gravitational interactions which are needed for them to have any cosmological consequences, and this is already enough. Gravitational interactions allow the process of figure 1, in which a ghost pair and photon pair are spontaneously created from the vacuum. The phase space integral is divergent, indicating a catastrophic instability. The divergent nature of the instability can only be avoided if we impose a Lorentz noninvariant momentum space cutoff on the final state phase space (more on this later). Setting such a cutoff at the scale $\Lambda$, the creation rate is, on dimensional grounds,

$$
\Gamma_{0 \rightarrow 2 \gamma 2 \phi} \sim \frac{\Lambda^{8}}{M_{p}^{4}}
$$


We have neglected Bose enhancement from final state occupancy, an assumption we will verify a posteriori. Notice that this pathology exists independently of the question of classical stability of the ghost-gravity system, which has been considered in [5]. The existence of this process is model independent - it requires only the existence of a wrong sign, canonical kinetic term and gravitational interaction for the ghosts.

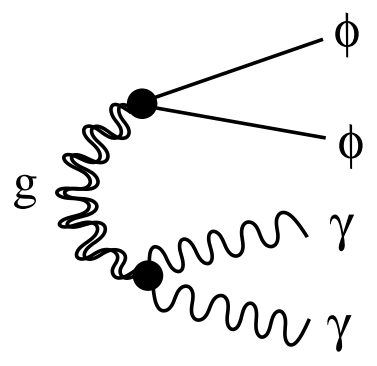

Figure 1. Graviton-mediated decay of vacuum into two ghosts and two photons.

An implicit excuse for even considering phantom matter at the classical level is perhaps the idea that, at long distances, the scalar field theory is merely an effective one, whose ultraviolet completion is well defined and respects unitarity. In this way, it might be possible to have a physical value for the cutoff in (3) which was small enough so that the rate of decay of the vacuum is slow on cosmological time scales. In this letter, we estimate just how low a cutoff $\Lambda$ is required for consistency with observational constraints. This question was previously considered in [6]; but we reach somewhat different conclusions, as we discuss below.

To find the density of photons which are spontaneously produced, we evolve the phase space density of ghosts and photons in an expanding universe,

$$
\frac{d}{d t}\left(a^{3} n\right)=a^{3} \Gamma
$$

where $a(t)$ is the scale factor and $\Gamma=\Gamma_{0 \rightarrow 2 \gamma 2 \phi}$. The solution is

$$
n(t)=\Gamma\left\{\begin{array}{ll}
\frac{t}{3 p+1}, & a(t) \sim t^{p} \\
H^{-1}\left(1-e^{-3 H t}\right), & a(t) \sim e^{H t}
\end{array} .\right.
$$

That is, the current number density is approximately given by the production rate per spacetime volume, $\Gamma$, times the age of the universe. Most of the photon pairs have been produced since redshift $z=1$, both because there has been more time since $z=1$ than before then, and because the density produced earlier was diluted by the expansion of the universe. This also means that their energy spectrum is not very different from the energy spectrum produced today; the spectrum peaks at $E \sim \Lambda$. Therefore we find,

$$
\frac{d n}{d E} \sim \Lambda^{7} M_{p}^{-4} t_{0} \quad \text { for } E \lesssim \Lambda
$$

This spectrum of photons with energy near $\Lambda$ is constrained by observations of the diffuse gamma ray background. EGRET [7] has measured the differential photon flux to be

$$
\frac{d F}{d E}=7.3 \times 10^{-9}\left(\frac{E}{E_{0}}\right)^{-2.1}\left(\mathrm{~cm}^{2} \mathrm{~s} \mathrm{sr} \mathrm{MeV}\right)^{-1}
$$

where $E_{0}=451 \mathrm{MeV}$. Demanding that (6) not exceed (7) gives the upper limit

$$
\Lambda \lesssim 3 \mathrm{MeV} .
$$

Since the observed gamma ray spectrum involves a mean particle occupancy which is orders of magnitude less than 1 , neglect of Bose stimulation was entirely justified.

We emphasize that this bound depends only upon the ghost having at least a minimal coupling to gravity. The possible presence of other couplings can only strengthen the result. Nor does it depend on whether the ghost has a potential, so long as its mass is less than $\Lambda$. In models of phantom cosmology, the mass is taken to be of order the present Hubble scale, $10^{-33} \mathrm{eV}$, so this is not restrictive.

The process $0 \rightarrow 2 \gamma 2 \phi$ is not the only allowed one; we can consider also the production of neutrinos and of $e^{+} e^{-}$pairs. The neutrinos are hard to observe, so no good constraint arises there. The $e^{+} e^{-}$constraint may be more fruitful, but the existence of galactic and Earth magnetic fields makes it somewhat more difficult to relate an incident $e^{+}$flux to the extragalactic density. However, a sufficiently dense intergalactic $e^{+} e^{-}$plasma would lead to excessive rescattering of the cosmic microwave sky. This leads to the constraint $\Lambda \lesssim 40 \mathrm{MeV}$, still weaker than (8).

Let us compare our bound (8) to those which were obtained in ref. 6]. There it was argued that one can constrain $\Lambda<10^{-3} \mathrm{eV}$ by considering the process $\phi \rightarrow g \phi \phi$, where $g$ is a graviton. This bound is incorrect, however. First of all, it arose by considering the "decay" rate of a ghost at rest, and insisting that this be longer than the Hubble time to prevent the exponential runaway generation of ghosts. But the produced ghosts typically carry energies $\sim \Lambda$, so their decay rates are strongly time dilated. Demanding only that the time dilated decay rate be less than $1 / t_{0}$ gives $\Lambda<50 \mathrm{MeV}$. Second, the constraint arose by considering an interaction $\mathcal{L}_{\text {eff }}=\phi g^{\mu \nu} \partial_{\mu} \phi \partial_{\nu} \phi$. Not only is this interaction model dependent; it is actually an artifact of a noncanonical normalization of the $\phi$ field kinetic term, and can be removed by a field redefinition. Therefore, the proposed decay mechanism does not actually work. Diagrammatically, this is because the total amplitude is zero, as shown in figure 2. The naive contribution from the contact term is canceled by the three other diagrams, built from the $\phi \eta^{\mu \nu} \partial_{\mu} \phi \partial_{\nu} \phi$ vertex contained in the interaction, and the $g^{\mu \nu} \partial_{\mu} \phi \partial_{\nu} \phi$ vertex from the standard kinetic term. Thus the rate for $\phi \rightarrow g \phi \phi$ is zero. Ref. [6] then proceeds to obtain the bound $\Lambda<100 \mathrm{MeV}$ by requiring the decay $\phi \rightarrow 2 g 3 \phi$ to be slower than the present Hubble rate. But 
this bound is model dependent; it requires higher derivative Lagrangian terms. It also suffers from the same error of using at-rest decay rates, so the quoted bound is orders of magnitude too stringent.

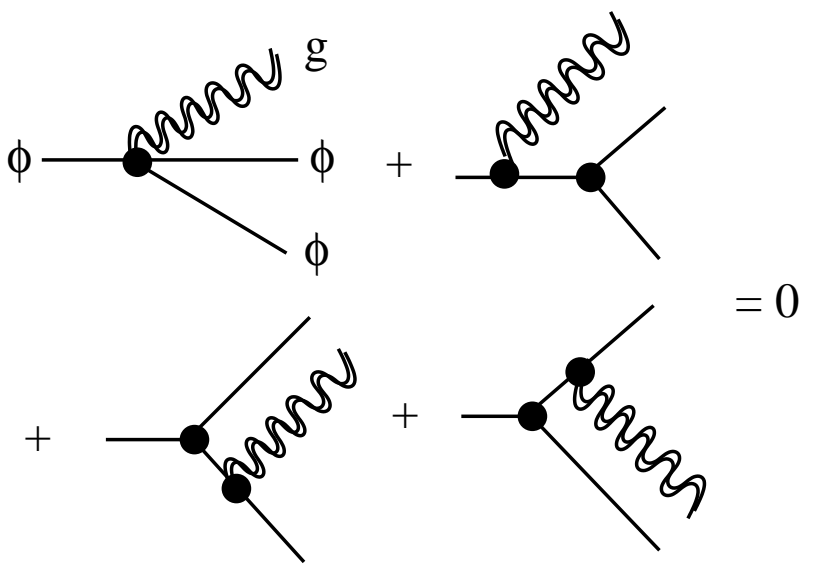

Figure 2. Vanishing of the amplitude for a ghost to decay into graviton and two ghosts.

Remarks. The stringent limit we have obtained from the diffuse gamma ray background, $\Lambda \lesssim 3 \mathrm{MeV}$, implies that any theory of low-energy effective ghosts must originate from new physics far below the TeV scale. Therefore we cannot invoke string theory, for example, as a plausible source for effective ghosts. Instead, we must imagine that they come from a low-energy sector that is completely hidden from the standard model, except for gravitational couplings. This makes ghosts look even more unlikely, in our view.

Another troubling feature is that, in order to pose this problem at all, we were forced to assume that Lorentz symmetry is broken. By taking the phase space for production of two photons plus two ghosts to be cut off at some momentum $\Lambda$, we have singled out a preferred frame, namely the rest frame of the cosmic microwave background radiation. Obviously there exist other frames where $k>\Lambda$ even if $k<\Lambda$ in the CMB rest frame. Such a cutoff might arise if, for example, the ghost dispersion relation had the form $\omega=-\sqrt{k^{2}-k^{4} / \Lambda^{2}}$, which would result from the Lorentz-violating Lagrangian $-\frac{1}{2}(\partial \phi)^{2}+$ $\frac{1}{2} \Lambda^{-2}\left(\vec{\nabla}^{2} \phi\right)^{2}$. The Lorentz-violating cutoff is necessary because, if we try to impose a Lorentz invariant cutoff, for instance on the virtuality of the off-shell graviton, then there is still a divergent integral over the boost, with respect to the microwave background frame, of the rest frame of the (timelike) graviton.

If we demand Lorentz invariance, but want to be maximally conservative, then we could argue that a process with a formation time longer than the age of the universe should not be considered. This places a bound on the boost between graviton and microwave frames, of $\gamma<t_{0} \sqrt{s}$, where $s$ is the Mandelstam variable (the 4momentum squared of the off-shell graviton). Imposing in addition the Lorentz invariant bound $s<\Lambda^{2}$ on the graviton propagator, the production rate becomes finite. Denoting the 4-momentum of the virtual graviton as $k$, the production rate is

$$
\Gamma \sim \int d^{4} k \theta\left(\Lambda^{2}-k^{2}\right) \theta\left(t_{0}-k_{0} / k^{2}\right) \frac{k^{4}}{M_{p}^{4}} \sim \frac{\Lambda^{10} t_{0}^{2}}{M_{p}^{4}}
$$

so that the number density is $\sim \Lambda^{10} t_{0}^{3} M_{p}^{-4}$. The typical energy of a produced photon is $k_{0} \sim \Lambda^{2} t_{0}$; even for a cutoff $\Lambda$ of order milli-electron volts, the energy is $\sim 10^{18}$ $\mathrm{GeV}$. The dominant mechanism by which gamma rays of such an energy scatter on the way to the Earth is $\gamma \gamma \rightarrow 4 e$, with the second $\gamma$ a microwave background photon; the free path is about 120 megaparsecs 8], leading to about a twenty-fold reduction in the flux. Arriving at the Earth, such a gamma ray would produce an air shower more energetic than any that have ever been seen. Using current bounds on the flux of such cosmic rays, less than 1 event per $\mathrm{km}^{2}$ per century [9], leads to a constraint of $\Lambda \lesssim 1 \mathrm{meV}$ (milli-electron volt). Gravity would receive order 1 modifications at a length scale $>0.2$ millimeters, in contradiction with experiment [10]. Hence, we conclude that even under very conservative assumptions, ghosts within a Lorentz invariant framework are experimentally excluded.

The requirement of Lorentz violation is worrisome, because it is inconsistent with general covariance. General covariance is the framework for general relativity, and it provides the gauge principle which guarantees the masslessness of the graviton. There are also very severe constraints on Lorentz violation within ordinary particle physics 11]; and Lorentz violation in another sector tends to be communicated to ordinary particle physics via graviton loops 12 . It is also troubling that, to our knowledge, no consistent construction of a low energy theory with ghosts from a ghost-free fundamental theory exists.

These considerations incline us toward the view that ghosts should not be feared, not because they are harmless, but because it is very unlikely that they exist. The inconveniences of a small cosmological constant seem much more bearable than those brought on by ghosts.

We thank Nima Arkani-Hamed, Ramy Brustein, Daniel Chung, Anne M. Green, Justin Khoury, Riccardo Rattazzi and Mark Trodden for useful remarks. JC acknowledges the Kavli Institute for Theoretical Physics and CERN theory group for their hospitality while this work was ongoing. We are supported in part by the Natural Sciences and Engineering Research Council of Canada and by le Fonds Nature et Technologies of Québec. S.J. also thanks RIKEN-BNL Center and U.S. Department of Energy [DE-AC02-98CH10886] for providing facilities essential for the completion of this work. 
[1] A. G. Riess et al. [Supernova Search Team Collaboration], "Observational Evidence from Supernovae for an Accelerating Universe and a Cosmological Constant," Astron. J. 116, 1009 (1998) arXiv:astro-ph/9805201.

S. Perlmutter et al. [Supernova Cosmology Project Collaboration], "Measurements of Omega and Lambda from 42 High-Redshift Supernovae," Astrophys. J. 517, 565 (1999) arXiv:astro-ph/9812133.

[2] C. L. Bennett et al., "First Year Wilkinson Microwave Anisotropy Probe (WMAP) Observations: Preliminary Maps and Basic Results," Astrophys. J. Suppl. 148, 1 (2003) arXiv:astro-ph/0302207.

[3] R. R. Caldwell, "A Phantom Menace?," Phys. Lett. B 545, 23 (2002) arXiv:astro-ph/9908168; A. E. Schulz and M. J. White, "The tensor to scalar ratio of phantom dark energy models," Phys. Rev. D 64, 043514 (2001) arXiv:astro-ph/0104112; J. g. Hao and X. z. Li, Phys. Rev. D 67, 107303 (2003) arXiv:gr-qc/0302100; G. W. Gibbons, "Phantom matter and the cosmological constant," arXiv:hep-th/0302199 X. z. Li and J. g. Hao, "O $(\mathrm{N})$ phantom, a way to implement $w<-1$," arXiv:hep-th/0303093 S. Nojiri and S. D. Odintsov, "Quantum deSitter cosmology and phantom matter," Phys. Lett. B 562, 147 (2003) arXiv:hep-th/0303117; S. Nojiri and S. D. Odintsov, "deSitter brane universe induced by phantom and quantum effects," Phys. Lett. B 565, 1 (2003) arXiv:hep-th/0304131; "Effective equation of state and energy conditions in phantom / tachyon inflationary cosmology perturbed by quantum effects," Phys. Lett. B 571, 1 (2003) arXiv:hep-th/0306212 ; P. Singh, M. Sami and N. Dadhich, "Cosmological dynamics of phantom field," Phys. Rev. D 68, 023522 (2003) arXiv:hep-th/0305110; J. g. Hao and X. z. Li, "Phantom with BornInfield type Lagrangian," Phys. Rev. D 68, 043501 (2003) arXiv:hep-th/0305207; "Constructing dark energy models with late time de Sitter attractor," Phys. Rev. D 68, 083514 (2003) arXiv:hep-th/0306033; M. P. Dabrowski, T. Stachowiak and M. Szydlowski, "Phantom cosmologies," arXiv:hep-th/0307128 D. j. Liu and X. z. Li, "Born-Infeld-type phantom on the brane world," Phys. Rev. D 68, 067301 (2003) arXiv:hep-th/0307239; L. P. Chimento and R. Lazkoz, "On the link between phantom and standard cosmologies," arXiv:gr-qc/0307111 E. Elizalde and J. Q. H, "Phantom and quantum matter in an anti-de Sitter universe," arXiv:gr-qc/0310128 H. Stefancic, "Generalized phantom energy," arXiv:astro-ph/0310904 V. B. Johri, "Phantom Cosmologies," arXiv:astro-ph/0311293

[4] S. Hannestad and E. Mortsell, "Probing the dark side: Constraints on the dark energy equation of state from $\mathrm{CMB}$, large scale structure and Type Ia supernovae," Phys. Rev. D 66, 063508 (2002)
arXiv:astro-ph/0205096; A. Melchiorri, L. Mersini, C. J. Odman and M. Trodden, "The State of the Dark Energy Equation of State," Phys. Rev. D 68, 043509 (2003) arXiv:astro-ph/0211522; J. A. S. Lima, J. V. Cunha and J. S. Alcaniz, "Constraining the dark energy with galaxy clusters X-ray data," Phys. Rev. D 68, 023510 (2003) arXiv:astro-ph/0303388.

J. L. Crooks, J. O. Dunn, P. H. Frampton, H. R. Norton and T. Takahashi, Astropart. Phys. 20, 361 (2003) arXiv:astro-ph/0305495.

[5] R. R. Caldwell, M. Kamionkowski and N. N. Weinberg, "Phantom Energy and Cosmic Doomsday," Phys. Rev. Lett. 91, 071301 (2003) arXiv:astro-ph/0302506; P. F. Gonzalez-Diaz, "You need not be afraid of phantom energy," Phys. Rev. D 68, 021303 (2003) arXiv:astro-ph/0305559; J. G. Hao and X. z. Li, "Phantom cosmic doomsday: a tale of two attractors," arXiv:astro-ph/0309746

[6] S. M. Carroll, M. Hoffman and M. Trodden, "Can the dark energy equation-of-state parameter $w$ be less than -1?," Phys. Rev. D 68, 023509 (2003) arXiv:astro-ph/0301273.

[7] P. Sreekumar et al., "EGRET observations of the extragalactic gamma ray emission," Astrophys. J. 494, 523 (1998) arXiv:astro-ph/9709257.

[8] R. J. Protheroe and P. L. Biermann, "A new estimate of the extragalactic radio background and implications for ultra-high-energy gamma ray propagation," Astropart. Phys. 6, 45 (1996) [Erratum-ibid. 7, 181 (1997)] arXiv:astro-ph/9605119.

[9] D. J. Bird et al. [HIRES Collaboration], "Evidence For Correlated Changes In The Spectrum And Composition Of Cosmic Rays At Extremely High-Energies," Phys. Rev. Lett. 71, 3401 (1993); "The Cosmic Ray Energy Spectrum Observed By The Fly's Eye," Astrophys. J. 424, 491 (1994).

[10] C. D. Hoyle, U. Schmidt, B. R. Heckel, E. G. Adelberger, J. H. Gundlach, D. J. Kapner and H. E. Swanson, "Submillimeter tests of the gravitational inverse-square law: A search for 'large' extra dimensions," Phys. Rev. Lett. 86, 1418 (2001) arXiv:hep-ph/0011014.

[11] S. R. Coleman and S. L. Glashow, "High-energy tests of Lorentz invariance," Phys. Rev. D 59, 116008 (1999) arXiv:hep-ph/9812418; V. A. Kostelecky and C. D. Lane, "Constraints on Lorentz violation from clock-comparison experiments," Phys. Rev. D 60, 116010 (1999) arXiv:hep-ph/9908504.

[12] C. P. Burgess, J. Cline, E. Filotas, J. Matias and G. D. Moore, "Loop-generated bounds on changes to the graviton dispersion relation," JHEP 0203, 043 (2002) arXiv:hep-ph/0201082. 